\title{
A Multicenter, Prospective Study of a New Fully Covered Expandable Metal Biliary Stent for the Palliative Treatment of Malignant Bile Duct Obstruction
}

\author{
Bret T. Petersen, ${ }^{1}$ Michel Kahaleh, ${ }^{2,3}$ Richard A. Kozarek, ${ }^{4}$ David Loren, ${ }^{5}$ Kapil Gupta, ${ }^{6,7}$ \\ Thomas Kowalski, ${ }^{5}$ Martin Freeman, ${ }^{8}$ Yang K. Chen, ${ }^{9}$ Malcolm S. Branch, ${ }^{10}$ \\ Steven Edmundowicz, ${ }^{11}$ Michael Gluck, ${ }^{4}$ Kenneth Binmoeller, ${ }^{12}$ Todd H. Baron, \\ Raj J. Shah, ${ }^{13}$ Timothy Kinney, ${ }^{14}$ William Ross, ${ }^{15}$ Paul Jowell, ${ }^{10}$ and David Carr-Locke ${ }^{16,17}$ \\ ${ }^{1}$ Charlton 8, GI Endoscopy, Mayo Clinic, 200 1st Street SW, Rochester, MN 55905, USA \\ ${ }^{2}$ Weill Cornell Medical College, 1305 York Avenue, 4th Floor, New York, NY 10065, USA \\ ${ }^{3}$ University of Virginia, Charlottesville, VA, USA \\ ${ }^{4}$ Virginia Mason Seattle Main Clinic, 1100 Ninth Avenue, Seattle, WA 98101, USA \\ ${ }^{5}$ Thomas Jefferson University, Main Building, Suite 480, 132 South 10th Street, Philadelphia, PA 19107, USA \\ ${ }^{6}$ Cedars-Sinai Medical Center, 8700 Beverly Bl No. 7511, Los Angeles, CA 90048, USA \\ ${ }^{7}$ Hennepin County Medical Center, Minneapolis, MN, USA \\ ${ }^{8}$ GI Division, Department of Medicine, Hennepin County Medical Center, MMC 36, 420 Delaware Street SE, \\ Minneapolis, MN 55455, USA \\ ${ }^{9}$ University of Colorado Boulder, Campus Box F735, Anschutz Inpatient Pavilion (AIP), S/M Gastroenterology, \\ Therapeutic Endoscopy, USA \\ ${ }^{10}$ Duke University, DUMC 3662, Durham, NC 27710, USA \\ ${ }^{11}$ Washington University, School of Medicine, 660 South Euclid Avenue, Campus Box 8124, St. Louis, MO 63110, USA \\ ${ }^{12}$ California Pacific Medical Center, 2351 Clay Street No. 304, San Francisco, CA 94115, USA \\ ${ }^{13}$ University of Colorado, Campus Box F735, Anschutz Outpatient Pavilion (AOP), Room 2136-A, \\ P.O. Box 6510, Aurora, CO 80045, USA \\ ${ }^{14}$ Hennepin County Medical Center, Medicine Office, GI 865B, 701 Park Avenue, Minneapolis, MN 55415, USA \\ ${ }^{15}$ The University of Texas MD Anderson Cancer Center, 1400 Pressler, Unit Number: Unit 1466, Houston, TX 77030, USA \\ ${ }^{16}$ Beth Israel Medical Center, 10 Union Square East, Suite 2G, New York, NY 10003, USA \\ ${ }^{17}$ Brigham and Women's Hospital, Boston, MA 02215, USA
}

Correspondence should be addressed to Bret T. Petersen; petersen.bret@mayo.edu

Received 19 September 2012; Accepted 22 January 2013

Academic Editor: Gianfranco D. Alpini

Copyright (C) 2013 Bret T. Petersen et al. This is an open access article distributed under the Creative Commons Attribution License, which permits unrestricted use, distribution, and reproduction in any medium, provided the original work is properly cited.

Background and Study Aims. Endoscopic placement of self-expanding metal stents (SEMSs) is indicated for palliation of inoperable malignant biliary obstruction. A fully covered biliary SEMS (WallFlex Biliary RX Boston Scientific, Natick, USA) was assessed for palliation of extrahepatic malignant biliary obstruction. Patients and Methods. 58 patients were included in this prospective, multicenter series conducted under an FDA-approved IDE. Main outcome measurements included (1) absence of stent occlusion within six months or until death, whichever occurred first and (2) technical success, need for reintervention, bilirubin levels, stent patency, time to stent occlusion, and adverse events. Results. Technical success was achieved in $98 \%$ (57/58), with demonstrated acute removability in two patients. Adequate clinical palliation until completion of followup was achieved in 98\% (54/55) of evaluable patients, with 1 reintervention due to stent obstruction after 142 days. Mean total bilirubin decreased from $8.9 \mathrm{mg} / \mathrm{dL}$ to $1.2 \mathrm{mg} / \mathrm{dL}$ at 1 month. Device-related adverse events were limited and included 2 cases of cholecystitis. One stent migrated following radiation therapy. Conclusions. The WallFlex Biliary fully covered stent yielded technically successful placement with uncomplicated acute removal where required, appropriate reduction in bilirubin levels, and low rates of stent migration and occlusion. This SEMS allows successful palliation of malignant extrahepatic biliary obstruction. 


\section{Introduction}

Endoscopic stent placement is now a standard therapy for management of most biliary strictures [1-3]. Plastic stents are generally employed in patients with benign or indeterminate strictures and for malignant strictures in patients with limited life expectancy [4]. Self-expanding metal stents (SEMSs) are indicated in patients with confirmed inoperable malignant biliary strictures. Bare SEMS are typically employed at the porta hepatis, while bare, partially covered, or fully covered SEMS may be used for extrahepatic lesions. Bare and partially covered SEMSs are prone to late occlusion due to ingrowth through the struts of the stent or overgrowth at the ends by tumor or hyperplastic tissue [5]. Some designs of partially and fully covered SEMS may be more prone to migration [6]. A fully covered stent with design features that limit migration yet facilitate repositioning or removal would provide optimal palliation of malignant strictures without significant risk related to inaccurate placement, size selection, or ultimate tissue diagnosis. The WallFlex Biliary RX Fully covered Stent is designed to meet these needs. This study was undertaken to evaluate the safety and clinical effectiveness of this new stent for palliative treatment in patients with inoperable extrahepatic malignant biliary obstruction.

\section{Patients/Materials and Methods}

This multisite, single arm, prospective study was performed at 10 centers under an Investigational Device Exemption (IDE) from the Food and Drug Administration (FDA). The study protocol and amendments were approved by Institutional Review Boards at all participating centers. All patients, or their legal representatives, provided written Informed Consent prior to enrollment. An Independent Medical Reviewer assessed events of stent occlusion or migration and all events resulting in death. This expert endoscopist was not an employee of the sponsor or involved with a participating center.

Inclusion criteria required (1) presence of inoperable malignant nonhilar extrahepatic biliary obstruction, (2) clinical symptoms of biliary obstruction, (3) age 18 or older, and (4) Willingness and ability to comply with study procedures and provide signed informed consent. Exclusion criteria included any of the following: (1) participation in another investigational study within 90 days prior to consent, (2) Strictures that could not be traversed by the delivery system, (3) perforation of any duct within the biliary tree, (4) presence of a biliary SEMS, (5) presence of any esophageal or duodenal stent, (6) contraindications to endoscopy, (7) sensitivity to any components of the stent or delivery system, (8) Active hepatitis, (9) intrahepatic metastases that extensively involve both lobes of the liver, and (10) life expectancy of $<3$ months.

The primary endpoint was adequate clinical palliation of biliary obstruction, as demonstrated by maintenance of bilirubin below $3 \mathrm{mg} / \mathrm{dL}$ or reduction by $>30 \%$ if the baseline value was greater than $3 \mathrm{mg} / \mathrm{dL}$ and absence of evidence of stent occlusion until death or 6 months of followup, whichever occurred first. A stent was considered occluded if the patient presented with elevation of total bilirubin that the treating physician deemed to be due to recurrent biliary obstruction and endoscopic imaging evidence of obstruction.

Secondary endpoints included (1) ability to deploy the stent in satisfactory position, (2) ability to successfully reposition or remove the stent, if required, at initial placement or at stent failure, without clinically significant complications or technical difficulties, (3) occurrence and severity of device and procedure-related adverse events, (4) need for reintervention, (5) reduction in symptoms of biliary obstruction (jaundice, pruritus, right upper quadrant pain, fever, nausea, vomiting, and dark urine) at all visits, (6) patency at 1,3 , and 6 months, and (7) time to stent occlusion.

2.1. Follow-Up Visits. Stent patency at 1 month was assessed by total bilirubin level and at 1,3, and 6 months by lack of obstructive symptoms. Follow-up visits were primarily conducted by phone, with inperson unscheduled visits required in case of recurrent obstructive symptoms.

2.2. Enrollment and Analysis Cohorts. A total of 74 patients who signed the informed consent form (ICF) were considered enrolled. After enrollment, fourteen patients failed screening relative to eligibility criteria and were withdrawn from the study without study treatment. Two patients were successfully treated with the study device but later found not to have met eligibility criteria. Per protocol, these patients were not part of the intent-to-treat (ITT) cohort, defined as those patients who signed the ICF, met eligibility criteria, and had the stent placement procedure initiated. The ITT cohort of 58 patients was used for assessment of baseline information and safety. Per protocol, assessment of the primary endpoint and secondary effectiveness endpoints was performed on the evaluable cohort, defined as ITT patients with at least one week of followup. Three patients were excluded from the evaluable cohort on the basis of death unrelated to the device prior to 1 week of followup (2) or failed placement of the study device at the index procedure with conversion to another device (1). Hence, the evaluable cohort had 55 patients.

2.3. Stent Design. The Wallflex Biliary RX fully covered stent is a radiopaque SEMS made of braided nitinol with a translucent silicone polymer (Permalume) lining of its entire length to prevent tumor or hyperplastic tissue ingrowth, while maintaining friction between stent wires and bile duct wall. The stent ends are flared to minimize risk of migration. Looped wires at the ends aim to minimize the risk of impaling or ulcerating the mucosa. The stent has a retrieval loop at the distal end to facilitate removal or repositioning in the event of misplacement or migration. The flared ends collapse when subjected to a withdrawal force. The stent is available in 8 and $10 \mathrm{~mm}$ diameters of varying lengths $(8 \mathrm{~mm} \times 60$ and $80 \mathrm{~mm}$, $10 \mathrm{~mm} \times 40,60$, and $80 \mathrm{~mm}$ ).

The delivery system is a coaxial tube design. The outer sheath constrains the stent before deployment and can be advanced to reconstrain the stent, if repositioning is necessary, after partial deployment down to the marked reconstrainment limit (approx. 80\% of the stent length). 
2.4. Statistics. A literature review conducted prior to the study noted that the majority $(\sim 80 \%)$ of publications on covered biliary SEMS report stent occlusion rates of $25 \%$ or less. Therefore, for this study, 55 evaluable patients were required to test whether the stent occlusion rate at 6 months or death was significantly less than $25 \%$, using a 1-sided binomial test with a significance level of $5 \%$ and a power of $90 \%$ for an expected stent occlusion rate of $10 \%$.

Kaplan-Meier analysis was used to determine the rates of survival and stent occlusion based on available followup information, with censoring for discontinued and deceased patients and beyond six months after stent placement. The number of biliary obstructive symptoms at each followup was summarized by the mean and its 95\% confidence interval, based on the normal distribution. A paired $t$-test was used to test the significance of reduction in the mean number of symptoms from baseline to 6 months, using the subset of patients with paired data available. Effectiveness measures were summarized by success rates and exact Clopper-Pearson $95 \%$ confidence intervals.

\section{Results}

The ITT cohort included 30 men and 28 women, with a mean age of 68.9 years (range 23-89.8). The top five reported biliary obstructive symptoms were jaundice $(82.8 \%)$, dark urine (37.9\%), right upper quadrant (RUQ) pain (34.5\%), pruritus $(32.8 \%)$, and nausea $(17.2 \%)$. Tumor type and stricture location are summarized in Tables 1 and 2. Twenty patients $(34.5 \%)$ had a previous biliary sphincterotomy.

3.1. Stent Placement and Removal. Stent placement (Figure 1) was technically successful in 57 of 58 patients (98\%) in the ITT cohort (Table 3). Deployment was attempted but failed in 1 patient due to severe angulation of the duodenoscope. In this patient the deployment system was removed and a noninvestigational biliary SEMS was placed during the index procedure.

Three stents were deployed but immediately removed from two patients to better match the pathologic anatomy. In one, a stent that was deemed to be of excessive length was deployed, but subsequently removed and replaced with a shorter sent. In the other patient, a $10 \mathrm{~mm} \times 60 \mathrm{~mm}$ stent proved insufficiently long and a $10 \mathrm{~mm} \times 40 \mathrm{~mm}$ stent placed through the initially placed stent did not expand completely within the tight stricture. Both were removed without technical or clinical complications, after which a $10 \mathrm{~mm} \times 80 \mathrm{~mm}$ stent was placed in good position. Thus, three stents were successfully removed without adverse events (Table 3 ). No patients underwent attempted stent removal after the initial stent placement procedure. All 57 ultimately placed study stents were $10 \mathrm{~mm}$ in diameter, with lengths of $40 \mathrm{~mm}$ in $14 \%$, $60 \mathrm{~mm}$ in $72 \%$, and $80 \mathrm{~mm}$ in $14 \%$ of stents.

3.2. Effectiveness. A summary of key effectiveness results is provided in Table 3. Overall 54 of 55 evaluable patients (98\%) had adequate clinical palliation of biliary obstruction until death or completion of followup. This rate of stent occlusion (2\%) was significantly less than those commonly noted in the
TABLE 1: Types of malignancy at baseline.

\begin{tabular}{lc}
\hline Cancer type & $\begin{array}{c}\text { ITT subjects } \\
(N=58 \text { subjects })\end{array}$ \\
\hline Pancreatic & $45(77.6 \%)$ \\
Cholangiocarcinoma & $3(5.2 \%)$ \\
Ampullary & $2(3.4 \%)$ \\
Colon & $2(3.4 \%)$ \\
Lung & $2(3.4 \%)$ \\
Other undefined malignancy & \\
$\quad$ Retroperitoneal mass, suspect sarcoma & \\
$\quad \begin{array}{l}\text { Malignant adenopathy of the porta hepatic } \\
\text { portacaval, and pericardial regions }\end{array}$ & $4(6.9 \%)$ \\
Peripancreatic malignant lymphadenopathy & \\
$\quad$ Lymphoma to porta hepatis & \\
\hline
\end{tabular}

TABLE 2: Baseline stricture locations.

\begin{tabular}{lc}
\hline Location of stricture & Baseline \\
& $N=58(\%)$ \\
\hline Distal common bile duct (CBD) & $41(70.7 \%)$ \\
Mid CBD & $9(15.5 \%)$ \\
Proximal common bile duct & $5(8.6 \%)$ \\
Distal CBD and mid CBD & $1(1.7 \%)$ \\
Junction of distal CBD and mid CBD & $1(1.7 \%)$ \\
Papilla & $1(1.7 \%)$ \\
\hline
\end{tabular}

literature $(25 \%)(P<0.0001)$. Of the 55 patients, 23 were followed to 6 months, 25 died while on study, and 7 were discontinued prior to month 6 or death. One patient with discontinued followup presented with bacteremia, cholangitis, and fever due to stent occlusion 142 days after stent placement. In this case, the distal end of the stent was collapsed on itself at the level of the papilla with repositioning wire tines extending from the papilla which detached during stent manipulation. The distal end of the stent was balloon dilated, and a commercial uncovered SEMS was placed inside the occluded stent with prompt clinical improvement. Another patient had an ampullary cancer and adequate clinical palliation, but experienced asymptomatic stent migration at day 84 , which was deemed a result of response to radiation therapy. The patient did not require restenting. Five additional patients were dropped from followup for reasons unrelated to the stent at $33,45,50,138$, and 161 days after placement, including 3 patients that underwent Whipple procedures despite not originally being considered surgical candidates, 1 patient categorized as lost to followup on day 138 despite multiple contact attempts after the week 1 visit when no biliary obstructive symptoms were reported, and 1 patient who withdrew consent early due to failing health.

Total bilirubin levels at baseline and month 1 were available for 45 of the 55 evaluable patients. Among them, $44 / 45$ (97.8\%) demonstrated successful maintenance of total bilirubin by the previously mentioned criteria, with the mean level reducing from $8.9 \mathrm{mg} / \mathrm{dL}$ (range $0.4-27.1$ ) to $1.2 \mathrm{mg} / \mathrm{dL}$ (0.2-8.0). One patient with underlying hepatitis $\mathrm{C}$ and known 


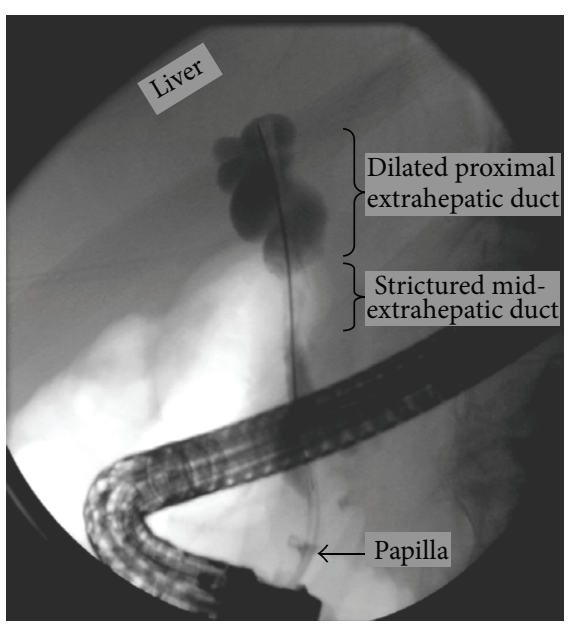

(a)

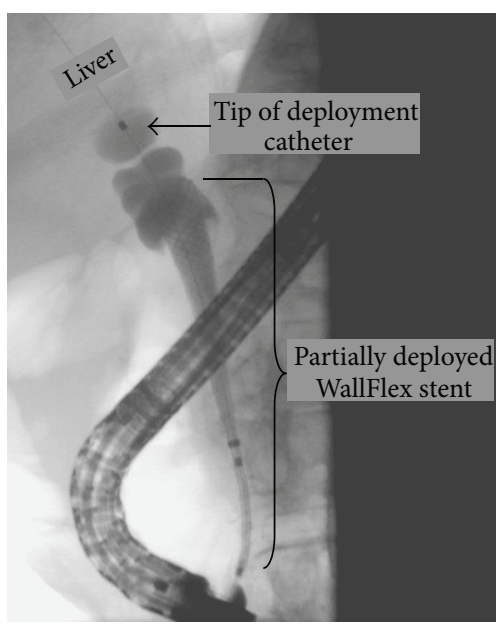

(b)

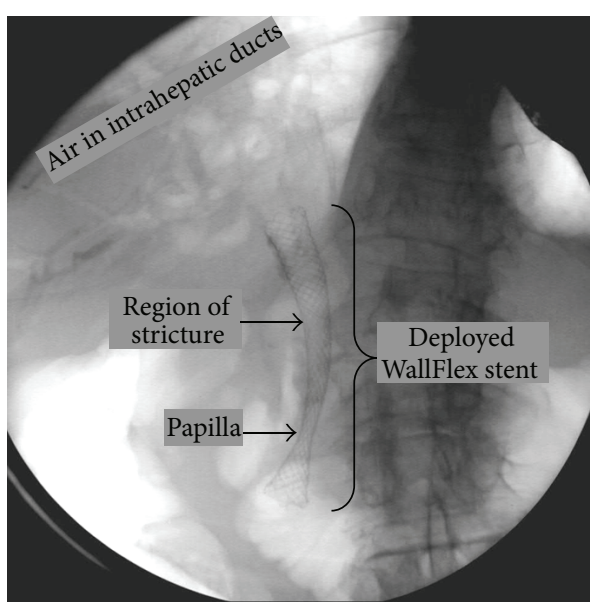

(c)

FIGURE 1: (a)-(c) Cholangiographic images of malignant biliary stricture and WallFlex Biliary Stent placement. (a) $254 \times 254 \mathrm{~mm}(96 \times$ $96 \mathrm{DPI})$, (b) $338 \times 338 \mathrm{~mm}(72 \times 72 \mathrm{DPI})$, and (c) $338 \times 338 \mathrm{~mm}(72 \times 72 \mathrm{DPI})$.

TABLE 3: Key effectiveness results.

\begin{tabular}{|c|c|c|}
\hline Measure & $\begin{array}{c}\text { Measure of } \\
\text { success } \\
N(\%)\end{array}$ & {$[95 \% \mathrm{CI}]$} \\
\hline \multicolumn{3}{|l|}{ Primary endpoint } \\
\hline Adequacy of palliation & $54(98.2 \%)$ & {$[90.3 \%, 100 \%]^{*}$} \\
\hline \multicolumn{3}{|l|}{ Secondary endpoints } \\
\hline $\begin{array}{l}\text { Stent deployment/technical } \\
\text { success }\end{array}$ & $57(98.3 \%)$ & {$[90.8 \%, 100 \%]$} \\
\hline \multicolumn{3}{|l|}{$\begin{array}{l}\text { Number of biliary obstructive } \\
\text { symptoms at baseline }\end{array}$} \\
\hline Mean $\pm \operatorname{SD}(N)$ & $2.7 \pm 1.76$ & {$[2.0,3.50]$} \\
\hline Range (Min, Max) & $(1,8)$ & \\
\hline Median & 2 & \\
\hline \multicolumn{3}{|l|}{$\begin{array}{l}\text { Number of biliary obstructive } \\
\text { symptoms at month } 6\end{array}$} \\
\hline Mean $\pm \mathrm{SD}(N)$ & $0.1 \pm 0.46$ & {$[0.00,0.33]$} \\
\hline Range (Min,Max) & $(0,2)$ & \\
\hline Median & 0 & \\
\hline Stent patency & $21(91.3 \%)$ & {$[72.0 \%, 98.9 \%]$} \\
\hline $\begin{array}{l}\text { Reduction of bilirubin level at } \\
\text { month } 1\end{array}$ & $44(97.8 \%)$ & {$[88.2 \%, 99.9 \%]$} \\
\hline
\end{tabular}

${ }^{*}$ Intent-to-treat (58).

cirrhosis failed to demonstrate successful reduction in total bilirubin at the month 1 visit (baseline total bilirubin = $11.0 \mathrm{mg} / \mathrm{dL}$; month $1=8.0 \mathrm{mg} / \mathrm{dL}$ ). In ten patients month 1 total bilirubin levels were not obtained due to early death (3) missing blood draw (3), early discontinuation (2), patient refusal (1), and loss to followup (1).

The mean number of reported biliary obstructive symptoms was reduced at each visit compared to the prior visit, ranging from a baseline mean for all 55 evaluable patients of $2.73 \pm 1.60$ (standard deviation (SD)) (range 1-8, median 2) to $0.13 \pm 0.46(\mathrm{SD})$ reported symptoms (range $0-2$, median 0 ) at

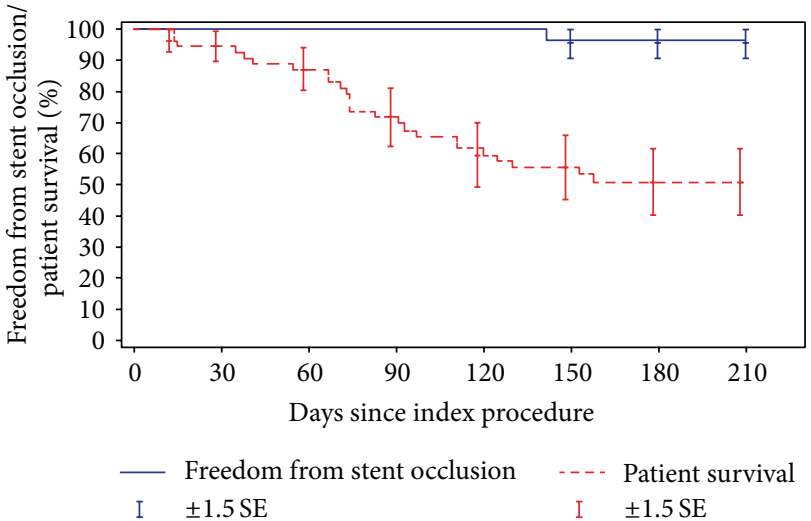

FIGURE 2: Kaplan-Meier curve of patient survival and freedom from stent occlusion $81 \times 40 \mathrm{~mm}(300 \times 300 \mathrm{DPI})$.

the month 6 visit. Analyzing the subset of 23 subjects with paired data available, there was a significant reduction in the mean number of symptoms from baseline to 6 months $(P<0.0001)$. By protocol criteria, stent patency was evident in $91.3 \%$ at Month 6.

The estimated patient survival rate and the estimated rate of freedom from stent occlusion, as a function of time after stent placement, are both demonstrated in Kaplan Meier curves in Figure 2. One patient (1.8\%) experienced a stent occlusion. Kaplan-Meier analysis based on the 55 evaluable patients yielded an estimated stent occlusion rate at 6 months of $3.7 \%$ (SD 3.6\%). The median time to stent occlusion is at least 210 days. Hence, the study stent appears effective in providing biliary obstruction-free palliation to most patients until death or at least 6 months after stenting.

3.3. Safety. There were no unanticipated adverse device effects (UADEs), and no deaths were attributed to the investigational device. In the ITT cohort, 4 patients experienced 6 serious adverse events (SAE's) deemed potentially (3) 
TABLE 4: Comparison to recent publications.

\begin{tabular}{|c|c|c|c|c|c|c|c|c|c|}
\hline First author & Enrollment & $\begin{array}{l}\text { Number of } \\
\text { centers }\end{array}$ & $\begin{array}{c}\text { Number of } \\
\text { patients }\end{array}$ & $\begin{array}{l}\text { Metal stent } \\
\text { type and } \\
\text { covering* }\end{array}$ & $\begin{array}{c}\text { Recurrent } \\
\text { biliary } \\
\text { obstruction rate }\end{array}$ & $\begin{array}{c}\text { Stent } \\
\text { patency at } \\
6 \text { months }\end{array}$ & $\begin{array}{l}\text { Migration } \\
\text { rate }\end{array}$ & $\begin{array}{l}\text { Cholecystitis } \\
\text { rate }\end{array}$ & $\begin{array}{c}\text { Pancreatitis } \\
\text { rate }\end{array}$ \\
\hline $\begin{array}{l}\text { Costamagna } \\
{[7]}\end{array}$ & 5 months & 6 & 66 & PC WallFlex & $6 \%(4)$ & $94 \%$ & $3 \%(2)$ & $5 \%(3)$ & $2 \%(1)$ \\
\hline \multirow[t]{2}{*}{ Isayama [8] } & 60 months & 4 & 138 & $\begin{array}{c}\text { PC } \\
\text { Wallstent }\end{array}$ & $21 \%(29)$ & - & $17 \%(24)$ & $7 \%(9)$ & $4 \%(5)$ \\
\hline & 11 months & 20 & 141 & PC WallFlex & $26 \%(36)$ & $78 \%$ & $8 \%(11)$ & $10 \%(14)$ & $6 \%(8)$ \\
\hline \multirow[t]{2}{*}{ Kullman [9] } & \multirow[t]{2}{*}{34 months } & \multirow[t]{2}{*}{10} & 200 & $\begin{array}{c}\text { UC } \\
\text { Nitinella }\end{array}$ & $23 \%(45)$ & $78 \%$ & $0 \%(0)$ & $1 \%(2)$ & $2 \%(4)$ \\
\hline & & & 200 & PC Nitinella & $24 \%(47)$ & $74 \%$ & $3 \%(6)$ & $1 \%(2)$ & $3 / 200$ \\
\hline Petersen & 6 months & 10 & 55 & FC WallFlex & $2 \%(1)$ & $91 \%$ & $2 \%(1)$ & $4 \%(2)$ & $2 \%(1)$ \\
\hline \multirow[t]{2}{*}{ Song [10] } & \multirow[t]{2}{*}{19 months } & \multirow[t]{2}{*}{1} & 24 & $\begin{array}{c}\text { Paclitaxel } \\
\text { covered } \\
\text { SEMS }\end{array}$ & $21 \%(5)$ & - & $0 \%(0)$ & $0 \%(0)$ & $4 \%(1)$ \\
\hline & & & 25 & FC SEMS & $32 \%(8)$ & - & $16 \%(4)$ & $0 \%(0)$ & $0 \%(0)$ \\
\hline Talreja [11] & 41 months & 6 & 260 & FC WallFlex & $3 \%(8)$ & $63 \%$ & $2 \%(4)$ & $1 \%(2)$ & $2 \%(4)$ \\
\hline \multirow{2}{*}{ Telford [12] } & \multirow{2}{*}{68 months } & \multirow{2}{*}{4} & 61 & $\begin{array}{c}\text { UC } \\
\text { Wallstent }\end{array}$ & $18 \%(11)$ & $90 \%$ & $0 \%(0)$ & $5 \%(3)$ & $2 \%(1)$ \\
\hline & & & 68 & $\begin{array}{c}\text { PC } \\
\text { Wallstent } \\
\end{array}$ & $29 \%(20)$ & $87 \%$ & $12 \%(8)$ & $4 \%(3)$ & $0 \%(0)$ \\
\hline Tringali [13] & 30 months & $\begin{array}{c}\text { Unknown } \\
\text { (multicenter) }\end{array}$ & 70 & PC ComVi & $30 \%(21)$ & $70 \%$ & $4 \%(3)$ & $1 \%(1)$ & $0 \%(0)$ \\
\hline
\end{tabular}

${ }^{*}$ FC: fully covered; PC: partially covered; UC: uncovered.

or probably (3) related to the study device. Cholecystitis occurred in 2 patients, 1 of whom also experienced gallbladder perforation. In this patient, purulent bile and gallstones were noted at the time of prior plastic stent removal during the index procedure. In both patients who experienced cholecystitis the stents were placed across the cystic duct; however, it is unknown whether there was tumor involvement at that level. Both events resolved with antibiotics and percutaneous gallbladder drain placement. The prevalence of intact gallbladders among treated patients was not systematically documented; hence the rate of acute cholecystitis among those with intact gallbladders is unknown. Post-ERCP pancreatitis occurred in one patient who later also developed self-limited RUQ pain. One patient experienced severe, selflimited postprocedural nausea. The SAE of cholangitis/stent occlusion at day 142 noted in the effectiveness section was reported by the investigator as related to progression of underlying disease rather than the stent. The stent migration reported in the effectiveness section was not associated with an adverse event as it did not lead to patient symptoms.

Six additional patients experienced a total of 7 SAEs reported as probably or possibly related to the ERCP itself, including one case of respiratory compromise during procedural sedation, culminating in death 8 days later from resulting sequelae and underlying pancreatic cancer, and one case each of post-ERCP pancreatitis and of postsphincterotomy bleeding, both of which resolved without sequelae. Individual cases of fever, abdominal pain, and nausea that may have been procedure related resolved without sequelae.

\section{Discussion}

Studies of endoscopic palliation for malignant inoperable obstructive jaundice have demonstrated the therapeutic advantage and cost effectiveness of self-expanding metal stents compared to surgical bypass or placement of plastic stents $[2,14,15]$. The efficacy of bare metal stents is limited by the propensity for tumor ingrowth [16]. Similarly, partially covered SEMSs remain prone to obstruction by ingrowth and overgrowth of tumor and by hyperplastic tissue response at the short bare segment at the upper end. Thus, the potential benefit of the central covering is offset by the persistent risk of occlusion at the uncovered ends [12, 17-19]. Fully covered designs without sharp wire ends may preclude these complications and thereby yield prolonged freedom from stent occlusion. In this series, only one patient experienced recurrent biliary obstruction, after 142 days.

Recurrent biliary obstruction caused by migration or stent occlusion remains the most important issue. It is still unproven whether CSEMS will be a better solution than uncovered SEMS as evidenced by 2 recently published randomized studies, $[9,12]$; however, a recent meta-analysis [20] showed significantly prolonged stent patency and stent survival for covered stents. Data from this series and Costamagna et al. [7] suggest that the covered WallFlex stent design may lead to a lower recurrent biliary obstruction rate compared to older designs (Table 4). Despite the full length covering on the WallFlex stent used in the present series, only one stent migrated, and this was asymptomatic in a patient 
with presumed stricture improvement following radiation therapy. Stent replacement was not required.

This study of a new fully covered SEMS demonstrates successful endoscopic palliation of unresectable malignant distal biliary obstruction in $98 \%$ of patients, suggesting that the goals of various stent design features were mostly met. As in most studies of biliary SEMS, technical placement success was almost universal. One stent failed to deploy adequately and was immediately replaced by a commercially available stent. Two patients' stents were incorrectly sized and therefore removed and replaced with different lengths. The narrow mesh design of the Wallflex stent foreshortens during placement and requires some attention to placement, compared to the wider mesh nonforeshortening laser cut designs.

For practical purposes bare SEMSs are not removable following the procedure at which they were inserted. Although there are descriptions of successful removal of uncovered biliary stents, it is technically difficult to perform and not predictable [21]. The partially and fully covered metal stents do not carry labeling for removability in the USA; however, they have been employed "off-label" for limited durations in various nonmalignant conditions such as benign strictures $[22,23]$ and intractable bile leaks $[23,24]$. The partially covered versions are still prone to proximal ingrowth and potentially difficult removal $[25,26]$. This limits their use in benign applications, and some authors consider them inappropriate for use in patients with a life expectancy greater than 24 months [27]. In this study, we used a fully covered stent that is retained by collapsible flares at the ends, which make repositioning or immediate removal relatively atraumatic and highly predictable. In the porcine model this stent design was removed shortly after placement from all six animals without difficulty [28]. Similarly, in this study three stents were removed immediately without difficulty from two patients. If this ease of removal is confirmed in subsequent studies with longer indwell periods, this stent may alter the paradigm for palliation of indeterminate biliary strictures that are presumed to be malignant but are still undefined [21]. As reported by Siddiqui et al., [29] fully covered CSEMS can now be used as an initial intervention to relieve malignant biliary obstruction, even in patients whose surgical resectability status is uncertain; however, the use of the stent in such manner would be considered "off-label" in the USA. Placement prior to or at the time of biopsy and prior to assessment of operability could then proceed without concern about the risk for permanence in potentially benign lesions or in lesions likely to resolve with medical therapy, such as strictures related to lymphoma or IgG4-related disease. Of note in this series, three patients were originally considered inoperable but later went on to surgery and en bloc stent removal.

This study is limited by the lack of a comparison group. Also, 17 patients in this series died of their underlying disease within three months, limiting assessment of the duration of freedom from stent occlusion. Nevertheless, insertion success, duration of adequate clinical palliation, and complications were all superior or comparable to most published data on self-expanding biliary stents. Comparative trials of this fully covered stent versus noncovered or partially covered metal stents for extrahepatic malignant applications and versus multiple plastic stents for benign lesions are needed.

\section{Disclosure}

The following authors B. Petersen (Olympus America), M. Kahaleh (Boston Scientific, Fujinon, Emcision, MI TECH, Lumina, and Boston Scientific), R. Kozarek (Boston Scientific), S. Edmundowicz (Boston Scientific, Olympus America), T. Kowalski (Boston Scientific), R. Shah (Boston Scientific), D. Carr-Locke (US Endoscopy, Telemed, Boston Scientific, Cook Medical, Erbe USA, and BEE Corporation) disclosed financial relationships with commercial entities involved in gastrointestinal endoscopy.

\section{References}

[1] J. R. Andersen, S. M. Sorensen, A. Kruse, M. Rokkjaer, and P. Matzen, "Randomised trial of endoscopic endoprosthesis versus operative bypass in malignant obstructive jaundice," Gut, vol. 30, no. 8, pp. 1132-1135, 1989.

[2] E. L. A. Artifon, P. Sakai, J. E. M. Cunha et al., "Surgery or endoscopy for palliation of biliary obstruction due to metastatic pancreatic cancer," American Journal of Gastroenterology, vol. 101, no. 9, pp. 2031-2037, 2006.

[3] A. G. Speer, P. B. Cotton, and R. C. G. Russell, "Randomised trial of endoscopic versus percutaneous stent insertion in malignant obstructive jaundice," Lancet, vol. 2, no. 8550, pp. 57-62, 1987.

[4] M. J. Levy, T. H. Baron, C. J. Gostout, B. T. Petersen, and M. B. Farnell, "Palliation of malignant extrahepatic biliary obstruction with plastic versus expandable metal stents: an evidencebased approach," Clinical Gastroenterology and Hepatology, vol. 2, no. 4, pp. 273-285, 2004.

[5] M. Kahaleh, J. Tokar, M. R. Conaway et al., "Efficacy and complications of covered Wallstents in malignant distal biliary obstruction," Gastrointestinal Endoscopy, vol. 61, no. 4, pp. 528533, 2005.

[6] H. Isayama, Y. Komatsu, T. Tsujino et al., "A prospective randomized study of 'covered' versus 'uncovered' diamond stents for the management of distal malignant biliary obstruction," Gut, vol. 53, no. 5, pp. 729-734, 2004.

[7] G. Costamagna, A. Tringali, D. N. Reddy et al., "A new partially covered nitinol stent for palliative treatment of malignant bile duct obstruction: a multicenter single-arm prospective study," Endoscopy, vol. 43, no. 4, pp. 317-324, 2011.

[8] H. Isayama, T. Mukai, T. Itoi et al., "The final report of WATCH study; comparison of covered WallFlex stent with covered Wallstent as historical control by Japanese multicenter study in cases with malignant distal biliary obstruction," Gastrointestinal Endoscopy, vol. 76, no. 1, pp. 84-92, 2012.

[9] E. Kullman, F. Frozanpor, C. Sderlund et al., "Covered versus uncovered self-expandable nitinol stents in the palliative treatment of malignant distal biliary obstruction: results from a randomized, multicenter study," Gastrointestinal Endoscopy, vol. 72, no. 5, pp. 915-923, 2010.

[10] T. J. Song, S. S. Lee, S. C. Yun et al., "Paclitaxel-eluting covered metal stents versus covered metal stents for distal malignant biliary obstruction: a prospective comparative pilot study," Gastrointestinal Endoscopy, vol. 73, no. 4, pp. 727-733, 2011.

[11] J. P. Talreja, D. E. Loren, and T. E. Kowalski, "Evaluation of a fully covered self-expanding metal stent in malignant biliary 
obstruction: follow-up of a multi-center study," Gastrointestinal Endoscopy, vol. 73, no. 4, p. AB352, 2011.

[12] J. J. Telford, D. L. Carr-Locke, T. H. Baron et al., "A randomized trial comparing uncovered and partially covered self-expandable metal stents in the palliation of distal malignant biliary obstruction," Gastrointestinal Endoscopy, vol. 72, no. 5, pp. 907914, 2010.

[13] A. Tringali, M. Marchese, P. Familiari et al., "Self-expandable partially covered metallic stents to palliate malignant biliary strictures: evaluation of the ComVi stent," Gastrointestinal Endoscopy, vol. 71, no. 5, p. AB309, 2010.

[14] A. C. Moss, E. Morris, J. Leyden, and P. MacMathuna, "Do the benefits of metal stents justify the costs? A systematic review and meta-analysis of trials comparing endoscopic stents for malignant biliary obstruction," European Journal of Gastroenterology and Hepatology, vol. 19, no. 12, pp. 1119-1124, 2007.

[15] R. Watson, K. Bangatelos, J. W. Ostroff et al., "Comparison of metal stent placement following initial metal stent dysfunction in the palliation of malignant biliary obstruction," Gastrointestinal Endoscopy, vol. 71, p. AB301, 2011.

[16] J. N. Rogart, A. Boghos, F. Rossi et al., "Analysis of endoscopic management of occluded metal biliary stents at a single tertiary care center," Gastrointestinal Endoscopy, vol. 68, no. 4, pp. 676682, 2008.

[17] K. A. Hausegger, S. Thurnher, G. Bodendörfer et al., "Treatment of malignant biliary obstruction with polyurethane-covered Wallstents," American Journal of Roentgenology, vol. 170, no. 2, pp. 403-408, 1998.

[18] W. J. Yoon, J. K. Lee, K. H. Lee et al., "A comparison of covered and uncovered Wallstents for the management of distal malignant biliary obstruction," Gastrointestinal Endoscopy, vol. 63, no. 7, pp. 996-1000, 2006.

[19] D. H. Park, M. H. Kim, J. S. Choi et al., "Covered versus uncovered wallstent for malignant extrahepatic biliary obstruction: a cohort comparative Analysis," Clinical Gastroenterology and Hepatology, vol. 4, no. 6, pp. 790-796, 2006.

[20] A. Saleem, C. L. Leggett, M. H. Murad, and T. H. Baron, "Metaanalysis of randomized trials comparing the patency of covered and uncovered self-expandable metal stents for palliation of distal malignant bile duct obstruction," Gastrointestinal Endoscopy, vol. 74, no. 2, pp. 321-e27, 2011.

[21] B. T. Petersen, "SEMS removal: salvage technique or new paradigms," Gastrointestinal Endoscopy, vol. 62, no. 6, pp. 911-913, 2005.

[22] M. Kahaleh, B. Behm, B. W. Clarke et al., "Temporary placement of covered self-expandable metal stents in benign biliary strictures: a new paradigm? (with video)," Gastrointestinal Endoscopy, vol. 67, no. 3, pp. 446-454, 2008.

[23] J. A. Kasher, J. G. Corasanti, P. R. Tarnasky, L. McHenry, E. Fogel, and J. Cunningham, "A multicenter analysis of safety and outcome of removal of a fully covered self-expandable metal stent during ERCP," Gastrointestinal Endoscopy, vol. 73, no. 6, pp. 1292-1297, 2011.

[24] M. Kahaleh, V. Sundaram, S. L. Condron et al., “Temporary placement of covered self-expandable metallic stents in patients with biliary leak: midterm evaluation of a pilot study," Gastrointestinal Endoscopy, vol. 66, no. 1, pp. 52-59, 2007.

[25] P. Familiari, M. Bulajic, M. Mutignani et al., "Endoscopic removal of malfunctioning biliary self-expandable metallic stents," Gastrointestinal Endoscopy, vol. 62, no. 6, pp. 903-910, 2005.
[26] M. Kahaleh, J. Tokar, T. Le, and P. Yeaton, "Removal of selfexpandable metallic Wallstents," Gastrointestinal Endoscopy, vol. 60, no. 4, pp. 640-644, 2004.

[27] H. P. P. Siriwardana and A. K. Siriwardena, "Systematic appraisal of the role of metallic endobiliary stents in the treatment of benign bile duct stricture," Annals of Surgery, vol. 242, no. 1, pp. 10-19, 2005.

[28] B. T. Petersen, J. Damarati, and P. G. Gregory, "Acute removability study of the partially-covered Wallflex metal biliary stent in a porcine model," Gastrointestinal Endoscopy, vol. 65, no. 5, p. AB231, 2007.

[29] A. A. Siddiqui, V. Mehendiratta, D. Loren, S. K. Hong, and T. Kowalski, "Fully covered self-expandable metal stents are effective and safe to treat distal malignant biliary strictures, irrespective of surgical resectability status," Journal of Clinical Gastroenterology, vol. 45, no. 9, pp. 824-827, 2011. 


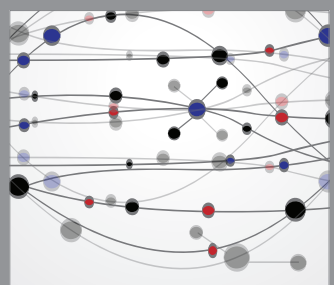

The Scientific World Journal
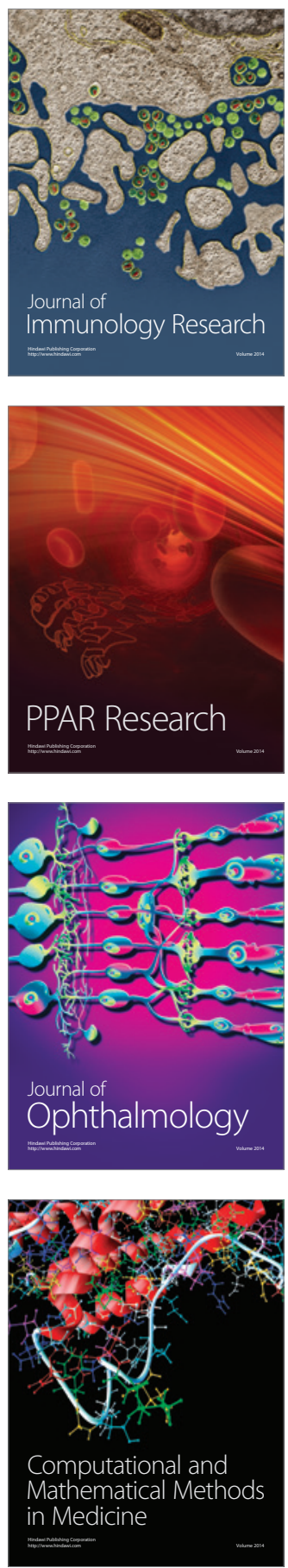

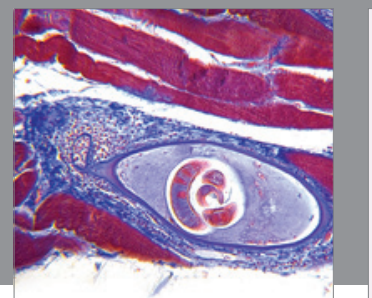

Gastroenterology

Research and Practice
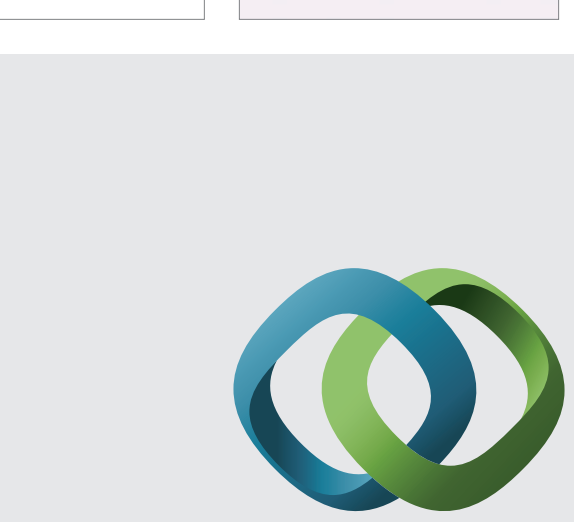

\section{Hindawi}

Submit your manuscripts at

http://www.hindawi.com
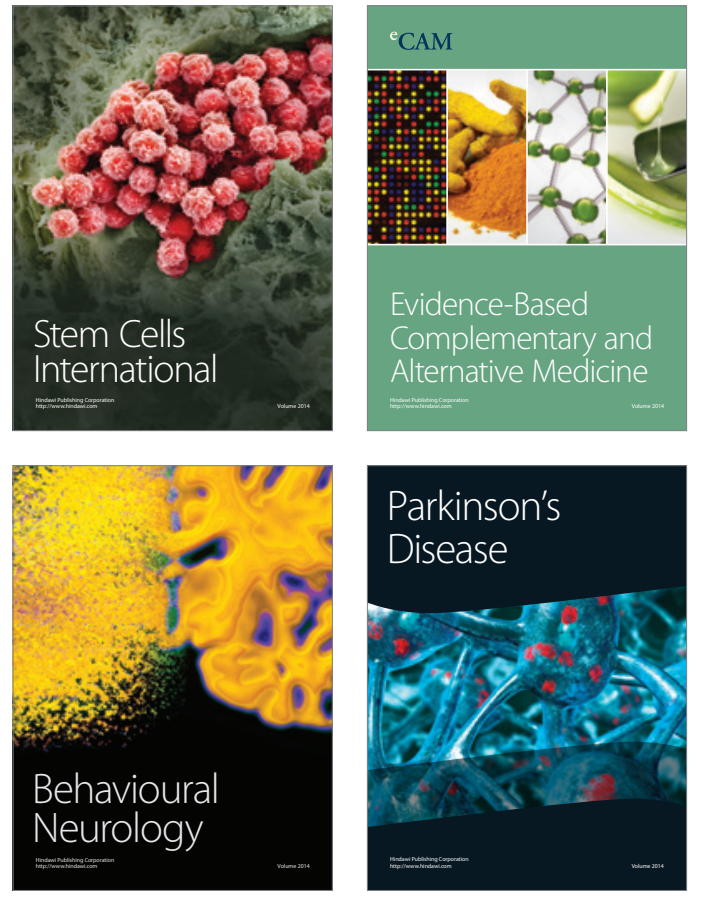
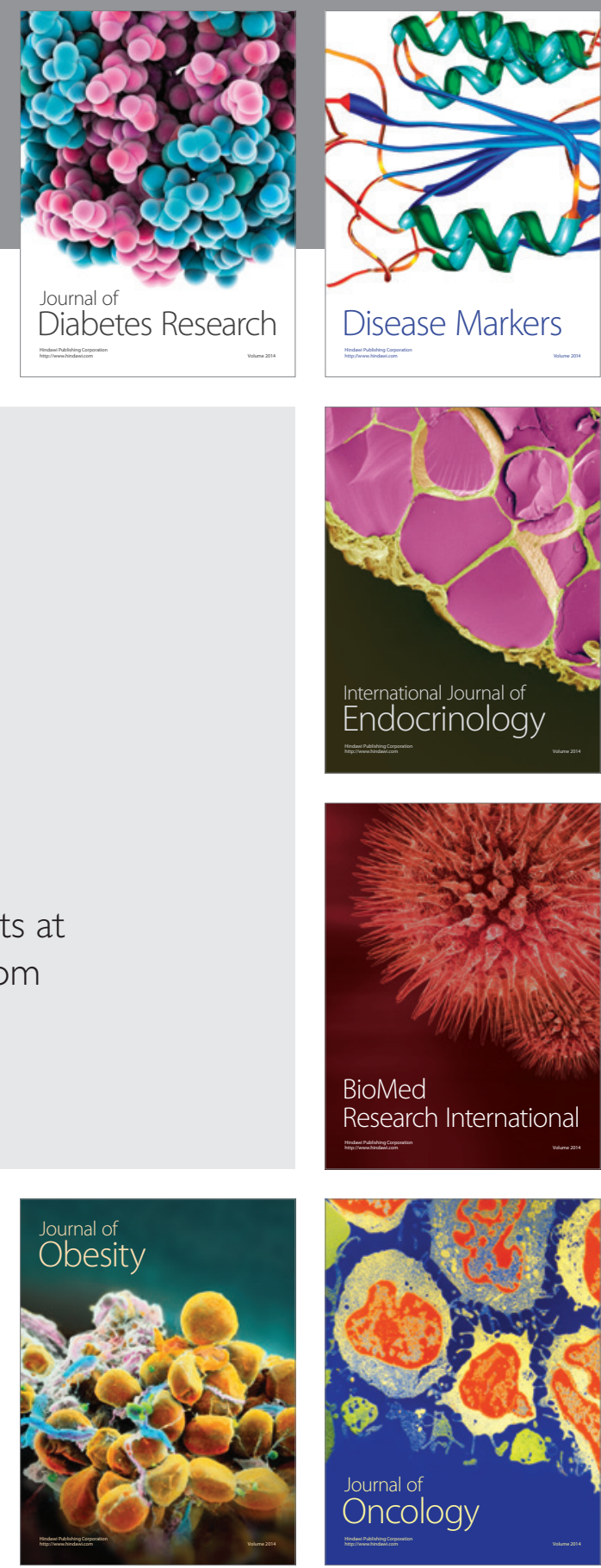

Disease Markers
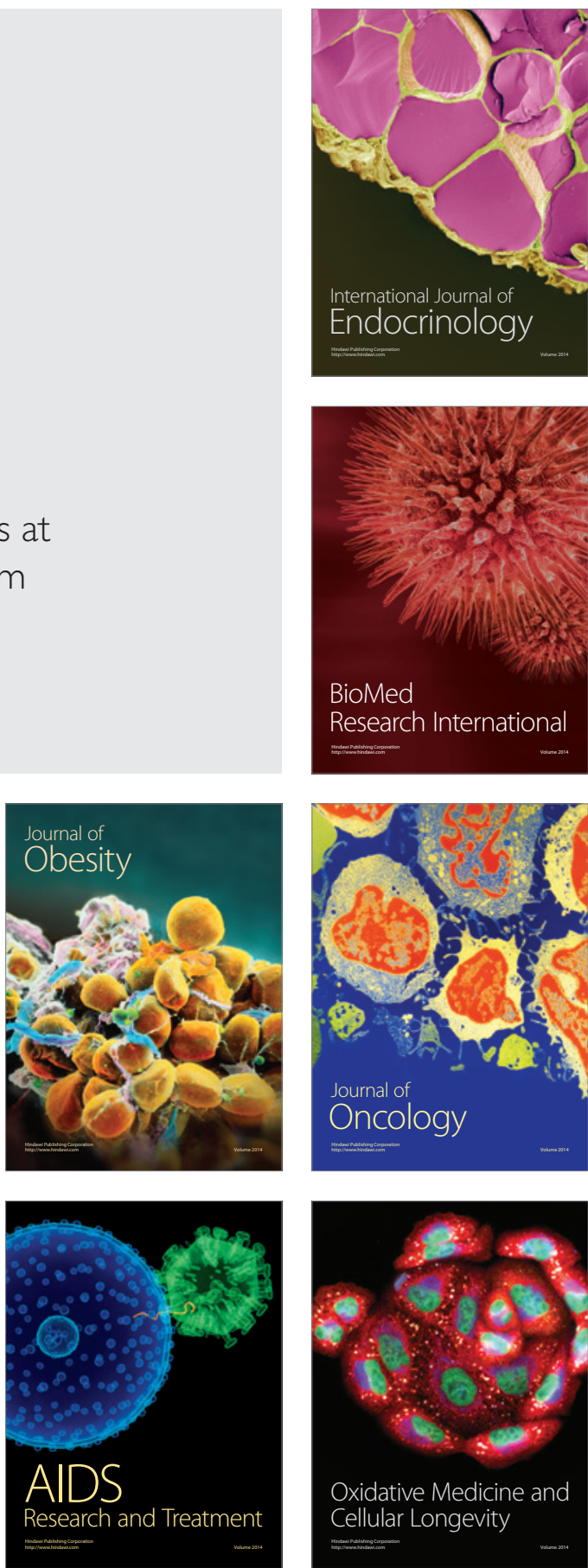